\title{
N ACM/SIGAI Autonomous Agents Research Award for Dr. Catherine Pelachaud
}

DOI: $10.1145 / 2757001.2757008$

The selection committee for the ACM/SIGAI Autonomous Agents Research Award is pleased to announce that Dr. Catherine Pelachaud, Di-

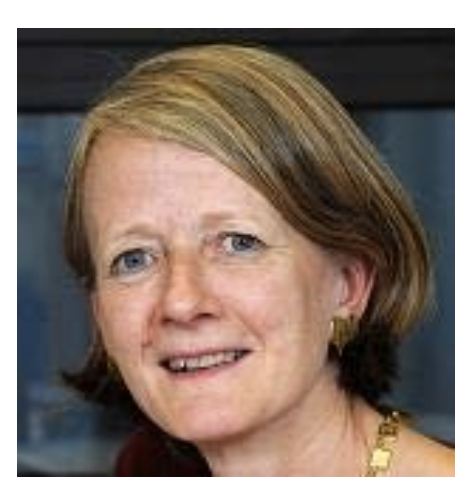

rector of Research at CNRS at Telecom ParisTech is the recipient of the 2015 award. Dr. Pelachaud is honored for her sustained and substantial contributions to the area of intelligent virtual agents. Her seminal work in this area helped launch the area of intelligent virtual agents, and her many subsequent publications have exerted a strong influence on virtual agent research at AAMAS and the Intelligent Virtual Agents conferences. Furthermore, her research on the role that bodily communication plays in face-to-face interaction has helped set the agenda of embodied agent research. In the process, it has firmly established a research area of modeling the body, its relation to the mind and its role in social interaction. Overall, this work has filled critical gaps in agents research often ignored by the larger community. 